\title{
Evaluation of Microstructure and Transport Properties of Deteriorated Cementitious Materials from Their X-ray Computed Tomography (CT) Images
}

\author{
Michael Angelo B. Promentilla ${ }^{1, *}$, Shermaine M. Cortez ${ }^{1}$, Regina Anne DC. Papel ${ }^{1}$, \\ Bernadette M. Tablada ${ }^{1}$ and Takafumi Sugiyama ${ }^{2}$ \\ 1 Chemical Engineering Department, De La Salle University, Manila 0922, Philippines; \\ cortezshayne@yahoo.com (S.M.C.); reginadcp21@gmail.com (R.A.D.P.); \\ bernadette.tablada@gmail.com (B.M.T.) \\ 2 Graduate School of Engineering, Hokkaido University, Sapporo 060-0808, Japan; takaf@eng.hokudai.ac.jp \\ * Correspondence: michael.promentilla@dlsu.edu.ph; Tel.: +63-02-536-0223 \\ Academic Editor: Hong Wong \\ Received: 14 February 2016; Accepted: 11 May 2016; Published: 19 May 2016
}

\begin{abstract}
Pore structure, tortuosity and permeability are considered key properties of porous materials such as cement pastes to understand their long-term durability performance. Three-dimensional image analysis techniques were used in this study to quantify pore size, effective porosity, tortuosity, and permeability from the X-ray computed tomography (CT) images of deteriorated pastes that were subjected to accelerated leaching test. X-ray microtomography is a noninvasive three-dimensional (3D) imaging technique which has been recently gaining attention for material characterization. Coupled with 3D image analysis, the digitized pore can be extracted and computational simulation can be applied to the pore network to measure relevant microstructure and transport properties. At a spatial resolution of $0.50 \mu \mathrm{m}$, the effective porosity $\left(\phi_{e}\right)$ was found to be in the range of 0.04 to 0.33 . The characteristic pore size $(d)$ using a local thickness algorithm was found to be in the range of 3 to $7 \mu \mathrm{m}$. The geometric tortuosity $\left(\tau_{g}\right)$ based on a 3D random walk simulation in the percolating pore space was found to be in the range of 2.00 to 7.45 . The water permeability values $(K)$ using US NIST Permeability Stokes Solver range from an order of magnitudes of $10^{-14}$ to $10^{-17} \mathrm{~m}^{2}$. Indications suggest that as effective porosity increases, the geometric tortuosity increases and the permeability decreases. Correlation among these microstructure and transport parameters is also presented in this study.
\end{abstract}

Keywords: 3D image analysis; X-ray microtomography; deteriorated cement paste; accelerated leaching; porosity; tortuosity; water permeability

\section{Introduction}

Portland cement-based concrete is one of the most widely used construction materials in infrastructure throughout the world [1]. However, one of the pressing issues faced by the construction industry is how to sustain durable service life performance during the design life of concrete structures. It has been recognized that the microstructure-transport property relationships of concrete play a key role in its durability, and therefore in its sustainability and high performance. For instance, water permeability is a good indicator of the durability of concrete particularly under coupled mechanical load and environmental factors [2,3]. Exposure to water during its service life is one of the primary causes of deterioration, as water percolates through an open crack or connected pore space and transports aggressive ions such as chlorides and sulfates. Moreover, progressive deterioration of concrete due to leaching also begins at the surface of the material where abrasion occurs when in contact with water for a long time, as seen in concrete used in dams, underground storage, and 
radioactive waste repository. The microstructure of cementitious matrix becomes more porous as leaching of calcium ions induces the dissolution of cement hydrates, and eventually leads to increased permeability [4] and degraded mechanical properties [5,6]. This slow leaching-induced degradation process opens up the material to even more water flow; hence, such vicious circles and synergistic interactions between leaching and other degradation mechanisms may speed up the deterioration and shorten the service life of concrete structures.

In general, the calcium leaching process is mainly controlled by two processes, namely the calcium dissolution from the cement hydrates in the solid matrix and the transport of these calcium ions in pore solutions $[7,8]$. As deterioration due to leaching is reported to be a very slow process [9], experimental studies such as the electrochemical-based accelerated leaching test have been conducted to understand the long-term degradation of cementitious materials $[10,11]$. Numerous studies have also been done to model the process as laboratory experiments are typically difficult and time consuming [12-14]. More recently, X-ray computed microtomography (XRCT), a nondestructive 3D imaging technique, has been employed to study the microstructure of these deteriorated cementitious materials as leaching progresses [15-17].

Although research has shown the changes in both the microstructural and macroscopic properties of cementitious materials with the progression of the leaching process, microstructure and transport parameters including water permeability were seldom quantified in the published literature. This is not surprising as it is difficult to visually examine the pore system of deteriorated cementitious materials during the leaching process and conduct tests without destroying the specimen while evaluating macroscopic properties such as water permeability. However, there have been recent studies in modeling water permeability and ionic diffusivity using lattice Boltzmann computations in a simulated microstructure [18,19]. Digitized images of the microstructure of cementitious materials from scanning electron or $\mathrm{X}$-ray tomographic microscopy have also been used to predict transport properties such as permeability $[20,21]$ and formation factor $[22,23]$. Thus, the objective of this exploratory study is to extend the work done in [16] by evaluating the microstructure-transport property relationships from the XRCT images of leaching-induced deteriorated cement pastes through 3D image analysis and computational simulation. A non-dimensional mathematical model was then developed to describe the relationship between the microstructure and transport properties of deteriorated cement paste, particularly the effective porosity, characteristic pore size, tortuosity, and intrinsic permeability.

\section{Methodology}

\subsection{Image Acquisition of Leaching-Induced Deteriorated Cement Pastes}

The computed tomography (CT) images used in this study were obtained from synchrotron-based X-ray computed microtomography (XRCT) of deteriorated cement paste [16]. For details of the experimental set-up and preparation of leaching-induced deteriorated cement paste sample, please refer to [16]. The cement used was JIS R5210-type ordinary Portland cement (OPC) which is commercially available in Japan. Its chemical composition by mass percent is as follows: $67.8 \%$ of $\mathrm{CaO}, 21.3 \%$ of $\mathrm{SiO}_{2}, 3.80 \%$ of $\mathrm{Al}_{2} \mathrm{O}_{3}, 2.41 \%$ of $\mathrm{Fe}_{2} \mathrm{O}_{3}$, and $2.20 \%$ of $\mathrm{SO}_{3}$. The hardened cement paste specimen (water to cement ratio of 0.50 ) was cured for 20 weeks prior to the accelerated leaching tests. The specimen was then subjected to an accelerated leaching test (see Figure 1) using electrochemical migration that simulated the long-term degradation behavior of the cement matrix. The electrochemical migration set-up consists of two compartments containing deionized water with a 10-mm thick specimen placed in-between. One compartment has a stainless steel (SUS) cathode and the other one is an anode made of platinum (Pt). An electrical DC power source which connects these two electrodes provides the potential gradient $(10$ volts $/ \mathrm{cm})$ across the specimen to force ionic migration. Thus, the $\mathrm{Ca}^{2+}$ ions move rapidly to the cathode side with the application of electrical field and this leads to the acceleration of the dissolution of hydrated cement products such as portlandite $(\mathrm{CaOH})$ and calcium silicate hydrates [10]. In this forced ionic migration test, cations in the pore solution such as $\mathrm{Na}^{+}, \mathrm{H}^{+}$, 
and $\mathrm{Ca}^{2+}$ move toward the cathode, as opposed to anions such as $\mathrm{OH}^{-}$and $\mathrm{SO}_{4}{ }^{2-}$ which move toward the anode side. Such ionic migration causes accelerated hydrolytic degradation of the cement paste leading to changes in microstructure, particularly for the surface in permanent contact with water near the electrodes. Note that the ions are equally transported in two directions toward the surface in contact with water through diffusion, but due to the effect of the imposed electric field, the movement of positively charged ions such as calcium ions toward the cathode accelerates.

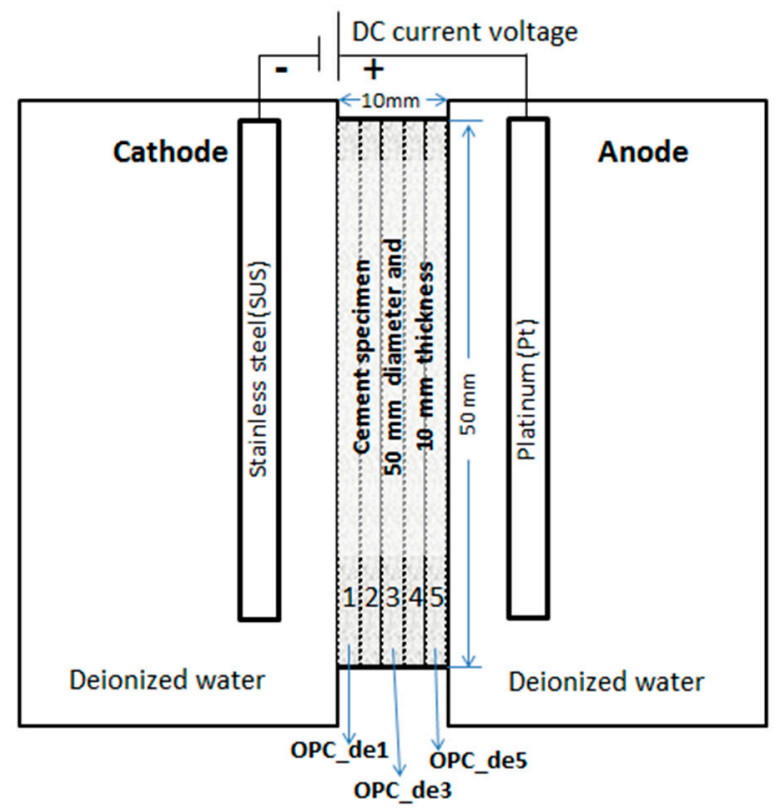

Figure 1. Schematic diagram of the accelerated leaching test of cement paste and regions of interest where 5 specimens were obtained (OPC_de1, OPC_de2, OPC_de3, OPC_de4, and OPC_de5).

However, in a natural leaching process, the $\mathrm{Ca}^{2+}$ and $\mathrm{OH}^{-}$from dissolution and decalcification of hydrated cement products move in the same direction under the diffusive transport mechanism. In the experimental test, $\mathrm{OH}^{-}$ions move in the opposite direction as compared with that of natural condition. In addition, the small current in the test induces water electrolysis forming $\mathrm{H}^{+}$and $\mathrm{O}_{2}$ in the anode side, and $\mathrm{OH}^{-}$and $\mathrm{H}_{2}$ in the cathode side, which does not occur under natural conditions. This may affect the ion transport efficiency and solubility because of the different $\mathrm{pH}$ condition. Nevertheless, several studies [9-11] demonstrated that the application of an electrical field leads to accelerated degradation of the cement paste following the same, or similar, mechanisms in natural scenarios.

In this study, the hardened (OPC) cement pastes were subjected to the said accelerated leaching test for 13 weeks prior to microtomographic examination. As shown in Figure 1, five samples from different regions of the cement matrix (OPC_de1,OPC_de2,OPC_de3,OPC_de4, and OPC_de5) were obtained and a shard from these samples was prepared for X-ray tomographic microscopy. The three dimensional (3D) image acquisitions were obtained from synchrotron-based XRCT facility at SPring-8, Japan (BL20XU, Harima Science Park City, Hyogo, Japan). In principle, X-ray microtomography is similar to medical computed tomography (CT) scanners in mapping the variation of the X-ray attenuation coefficient within the object and creating cross-sectional images but with higher spatial resolution. Since the X-ray attenuation coefficient depends on the atomic composition and density, the CT technique thus provides 3D grayscale images of the internal structure of the specimen without the laborious sectioning and sample preparation typically required in two-dimensional X-ray microscopy techniques. As long as the spatial resolution could be increased with respect to the microstructural feature of interest, the volumetric image obtained from these measurements could provide valuable 3D structural information. 
The spatial resolution of the reconstructed 3D images is 0.50 micrometers. In other words, this is the digitized image resolution that corresponds to the scanning isotropic voxel size of $0.50 \mu \mathrm{m}$. This scanning voxel size is the measure of the quality of the raw data CT images and determines the best level of detail that can be resolved in the image. Note that the digitized image obtained from $\mathrm{CT}$ is not just an image but a three-dimensional mapping of the linear attenuation coefficient (LAC) of the component in that voxel as being imaged in terms of gray scale value (GSV). This GSV is directly proportional to LAC as described in [23]. However, aside from the inherent statistical noise, partial volume effect or averaging is an image artifact that can be encountered in CT image analysis as the measured LAC depends on the restricted voxel size. These partial volume voxels represent a combination of materials due to the finite resolution of the imaging process, which yields a GSV representative of the average attenuation of these materials within a voxel. Thus, features such as finer pores with a size of $0.50 \mu \mathrm{m}$ or smaller cannot be detected or resolved properly. For example, if an isotropic voxel of $0.50 \mu \mathrm{m}$ comprises of smaller pores and other materials such as hydrated cement products, the resulting GSV (or measured LAC) represents some average property. Blurring at the interface of solid and pore also indicates that the voxel can be affected by the voxel of the surrounding area. In this regard, the selection of GSV for pore threshold value to extract the pore from the solid matrix is a non-trivial matter and still an open discussion. This is particularly true and challenging when the microstructure of interest is relatively small in relation to the resolution of the image acquisition.

\subsection{Evaluation of Microstructure and Transport Properties from CT Images}

From the reconstructed 3D-image data set, cubic volume of interest (VOI) of $300^{3}$ voxels was extracted. Five VOIs from each data set were randomly selected totaling of 25 VOIs for the 5 samples of deteriorated cement pastes. Porosity, pore size, geometric tortuosity, and permeability were analyzed for each VOI. Directional tortuosity and permeability in three orthogonal directions $(x, y$ and $z)$ were also computed, resulting to 75 data points.

Note that it is recognized that most studies on model cement pastes suggested that ordinary cement paste can be considered isotropic on a sufficiently high level of the microstructure $[24,25]$. However, there have been some studies indicating some degree of anisotropy in microstructure, particularly from images obtained from X-ray microtomography [22,26,27]. It is also imperative to understand the presence of structural anisotropy in damaged cement matrix as discussed in [28-30]. In this study, it is assumed that the local heterogeneity within the specimen may induce such structural anisotropy, given the nature of deterioration through the progression of leaching front toward the center or away from the surface in contact with water.

\subsubsection{Porosity and Mean Pore Size}

Quantification of porosity, which is the fraction of void space in the material, was done using the method described in [23]. The objective of the image analysis is to measure the segmented porosity and extract the largest percolating pore space from the volume of interest (VOI). It is important to determine this largest percolating pore cluster in any one of the orthogonal directions as this represents the effective (accessible) porosity for transport. In the absence of percolation, any microstructure-transport property relationship in the pore space would just be trivially meaningless. In this regard, segmentation and multiple cluster labeling techniques are used to identify the percolating pore space, if any, from the CT images.

Segmentation or image thresholding is the process of converting the gray scale CT image to a binary image by identifying the pore space and solid matrix based on their voxel intensity values (GSV). Segmented total porosity can then be measured by dividing the number of pore voxels with the total number of voxels in the VOI $\left(300^{3}\right.$ voxels). The segmentation method used for this study is a simple thresholding technique wherein the lower bound of gray scale value (GSV) associated with pore voxels was set to 0 while the upper bound is set to a pre-defined pore threshold value [23]. The pore threshold 
value was selected on the basis of transition point in segmented porosity-threshold dependency curve derived from the stack histogram of the 3D image dataset. At this transition point, it is assumed that the segmented porosity started to increase rapidly such that the boundary between pore and the solid matrix is most likely to be segmented as pore space. Note that this thresholding method is analogous to "overflow pore segmentation method" used for backscattered electron (BSE) images of cement-based materials described in [31]. Such a method was found to be more consistent and reliable than the other existing method for pore segmentation used in BSE images of cement-based material.

After segmentation, multiple cluster labeling was then employed to identify individual connected pore voxels and provide each with a unique label. Labeling indicates that any pore cluster with a distinct label is disconnected from any other pore clusters in the binary image. The algorithm described in [32] for multiple cluster labeling technique was used with the 6-point connectivity rule. Using this rule, when a pore voxel is sharing a common face with another voxel, the two voxels are considered to be connected, whereas those pore voxels that are in contact only at the vertex or edge are considered to be disconnected. The effective porosity is then quantified by dividing the number of pore voxels in the largest percolating pore cluster by the total number of voxels in VOI. ImageJ [33] and SLICE [34] were used to quantify and visualize both the segmented total porosity $(\psi)$ and effective porosity $\left(\phi_{\mathrm{e}}\right)$ from the CT images as shown in Figures 2 and 3. ImageJ, a public domain program which is extensible via plugins and a built-in macro interpreter, can do direct three dimensional analyses. For example, its BoneJ plugin can be used for three-dimensional study of the morphology, topology and texture together with advanced 3D visualization [35]. In addition, ImageJ was used for image denoising which applies algorithms such as the median filter or anisotropic diffusion filter to reduce noise while preserving the edges, lines or other details that are important for the interpretation of the image [36]. Such denoising is typically used as a pre-processing stage for segmentation in particular to image smoothing, edge detection and noise removal. Prior to 3D visualization via ImageJ, SLICE was also used for multiple cluster labelling of pore clusters to extract the effective porosity, i.e., the largest percolating pore cluster in the VOI.
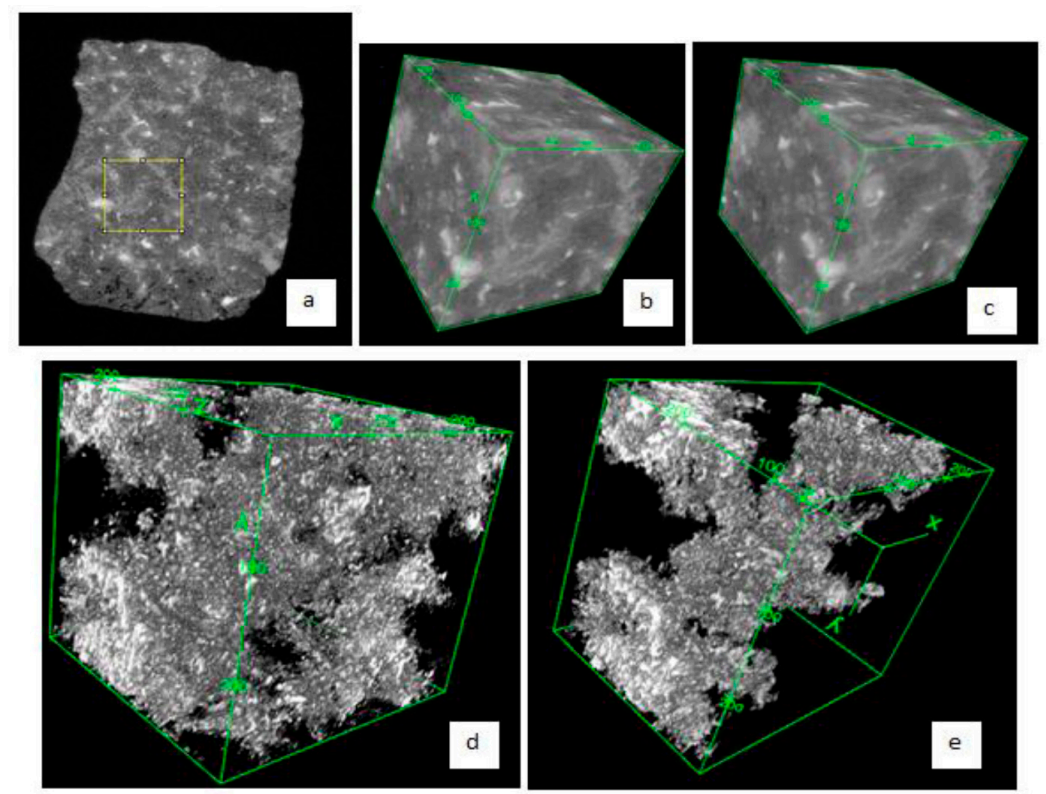

Figure 2. Extraction and visualization of pore space from CT images of deteriorated cement pastes: (a) An 8-bit representative slice $(2000 \times 2000$ voxels) obtained from OPC-de2; (b) The volume of interest $\left(300^{3}\right.$ voxels) obtained from the normalized data set; (c) The resulting 3D stack after denoising; (d) A 3D visualization of the segmented total porosity of the VOI; (e) A 3D visualization of the largest percolating pore cluster in the VOI or the effective porosity of the VOI. 


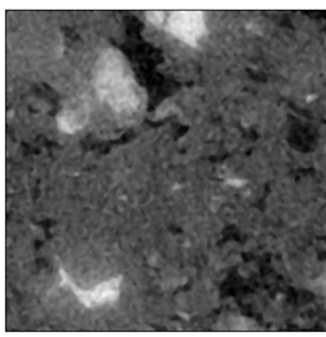

(a)

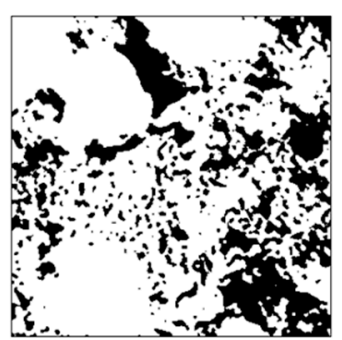

(b)

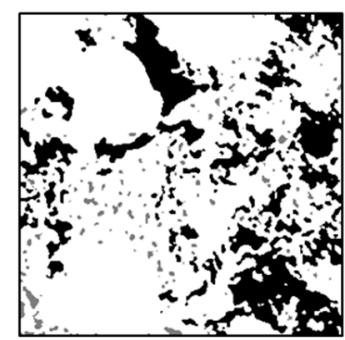

(c)

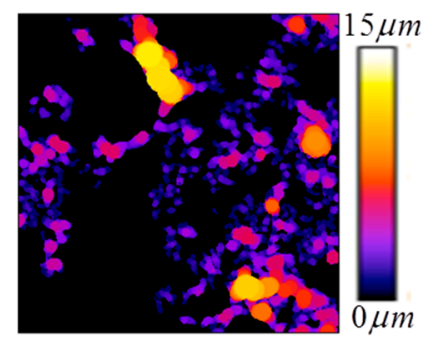

(d)

Figure 3. Image analysis for quantification of porosity and pore size: (a) An 8-bit representative grayscale slice $(300 \times 300$ voxels) from VOI; $(\mathbf{b})$ The binary image after segmentation (segmented porosity is imaged as black voxels); (c) The binary image after multiple cluster labeling (largest percolating pore cluster or effective porosity is imaged as black voxels whereas the smaller or isolated pore clusters are imaged as gray voxels); (d) Image resulting from local thickness algorithm to determine the mean pore size.

The characteristic pore size $(d)$ of the percolating pore cluster was determined using the maximal sphere algorithm as implemented in BoneJ plugin [35], which does not make any assumptions of underlying geometry to compute the mean thickness [37]. The pore size is analogous to the so-called model-independent "local thickness" which is a widely used parameter for morphometric analysis of trabecular bone structure, cellular solids, and paper fiber with a complex mixture of void space and solids [38], as well as for crack characterization in mortar and concrete [39,40]. The local thickness at any given point is defined as the diameter of the largest sphere that includes the point and can fit completely inside the structure. On the other hand, the mean thickness is computed from volume-average local thickness in the whole structure without assuming any structural type. Likewise, in describing the connected pore cluster as the structure of interest, one could define the size of a pore at any point within the pore space as the diameter of the largest sphere that includes this point and fits completely into the pore space. This maximal radius algorithm thus begins with the Euclidian Distance Transform method by calculating the metric distance of each solid voxel to the nearest solid (empty) space surface [38]. This distance is the radius of a sphere centered on this voxel that fits inside the pore structure. Redundant or smaller spheres are then eliminated producing a set of centers of maximal spheres filling the pore network completely. The local thickness or pore size for each portion of the structure is then twice the said radii of these spheres. The characteristic pore size is the corresponding volume-weighted mean pore size in the pore cluster. However, the smallest pore radius that can be included in the analysis is limited by the unit voxel size; hence, the accuracy of such parameter highly depends on the spatial resolution obtained from X-ray microtomography.

Sample slice output from the step-by-step image analysis is shown in Figure 3. In this figure, such pore size analysis is based on 3D algorithm but only 2D views in slice are shown for illustration. For example, the pore voxels, which are imaged as black in Figure $3 \mathrm{c}$ and as colored in Figure $3 \mathrm{~d}$, seem to be disconnected in 2D but are connected in three dimensions and part of the largest pore cluster.

\subsubsection{Tortuosity}

Tortuosity is a dimensionless parameter typically introduced as a fudge factor in transport equations to describe the influence of the complex morphology of pore structure on the transport properties of porous materials. The concept of geometric tortuosity $\left(\tau_{g}\right)$ introduced here is the ratio of the diffusion length $L_{D}$ and the geometric length $L$, i.e., the straight and shortest distance along the direction of the macroscopic flux:

$$
\tau_{g}=\frac{L_{D}}{L}=\sqrt{\frac{D_{o}}{D_{\infty}}}
$$


This geometric tortuosity can be quantified from the largest percolating pore space by computing the square root of the diffusion tortuosity derived from random walk simulations in a digitized pore network as described in [22,41]. This parameter includes not only the geometric details of the winding path but also the pore constriction and the nonuniform cross-sectional area of the pores. The diffusion tortuosity is defined as the ratio of the self-diffusion coefficient in the bulk medium (free space) to that of a confining geometry (connected pore space). Here $D_{0}$ refers to the time-independent self-diffusion coefficient of nonsorbing walkers in free space while $D_{\infty}$ is the limiting value of the self-diffusion coefficient in a well-connected pore space after a long diffusion time. With such a long time limit, the walkers fully experience the connectivity and high tortuosity in the system and the self-diffusion coefficient reaches a constant value. For unrestricted diffusion, $D_{0}=D(t)$. Thus, the diffusion tortuosity and the geometric tortuosity in free space are theoretically equal to one.

Note that the mean square displacement $\left\langle r^{2}(t)>\right.$ of a diffusing ion with self-diffusion coefficient $D$ in a background medium is governed by the Einstein-Smoluchowsky equation in $d$ dimensional space and as a function of time $(t)$ :

$$
<r^{2}(t)>=2 d \times D \times t
$$

The said equation provides the link between the macroscopic view of diffusion transport and the microscopic view of random walking non-sorbing ions [42]. Accordingly, the time-dependent self-diffusion coefficient of walkers is defined as follows:

$$
D(t)=\frac{1}{2 d} \frac{\mathrm{d}\left(\left\langle r^{2}(t)\right\rangle\right)}{\mathrm{dt}}
$$

Figure 4 illustrates the sample output if random walk simulation with 100,000 walkers is performed in free space ( $100 \%$ porosity) and in isolated and closed pore space. During the computational simulation, a number of walkers migrate on discrete voxels that correspond to pore space in a simple cubic lattice. A pore voxel is chosen randomly by each walker as the start position of the lattice walk time trial at $t=0$. A space step of one unit in one of the six possible directions is then performed as a trial move. After every random jump, $t$ is incremented by a unit integer time. If the said walker executes a random jump to one of the nearest pore voxels, the jump is performed, but if the randomly selected voxel is a solid voxel (such as those of voxel containing cement hydrates and anhydrous cement), the jump is not performed and time is still incremented. This describes the restricted motion analogous to that of the "blind ant trying to escape in the labyrinth" to simulate the diffusion of a particle in a disordered media as described in percolation theory [43]. In free space, the non-dimensional self-diffusion coefficient $\left(D_{0}\right)$ is one and it is time-independent as expected, whereas with a long diffusion time, this self-diffusion coefficient approaches zero in an isolated and closed pore space. Thus, the long-time behavior of the self-diffusion coefficient $\left(D_{\infty}\right)$ could approach a limiting value that is between zero and one as walkers probe the connectivity and tortuosity of an open pore network. The geometric tortuosity $\left(\tau_{g}\right)$ is then derived from the square root of the reciprocal of the normalized long-time self-diffusion coefficient obtained from random walk simulations in the pore network. Figure 5 describes a sample trajectory of a walker in a pore network in cement pastes. The computational time for random walk simulation in a VOI of $300^{3}$ voxels (e.g., in an Intel core i3 processor of $3.50 \mathrm{GHz}$ with $8 \mathrm{~Gb} \mathrm{RAM}$ ) is around two hours for lattice walk time steps of 500,000 with 100,000 walkers. 

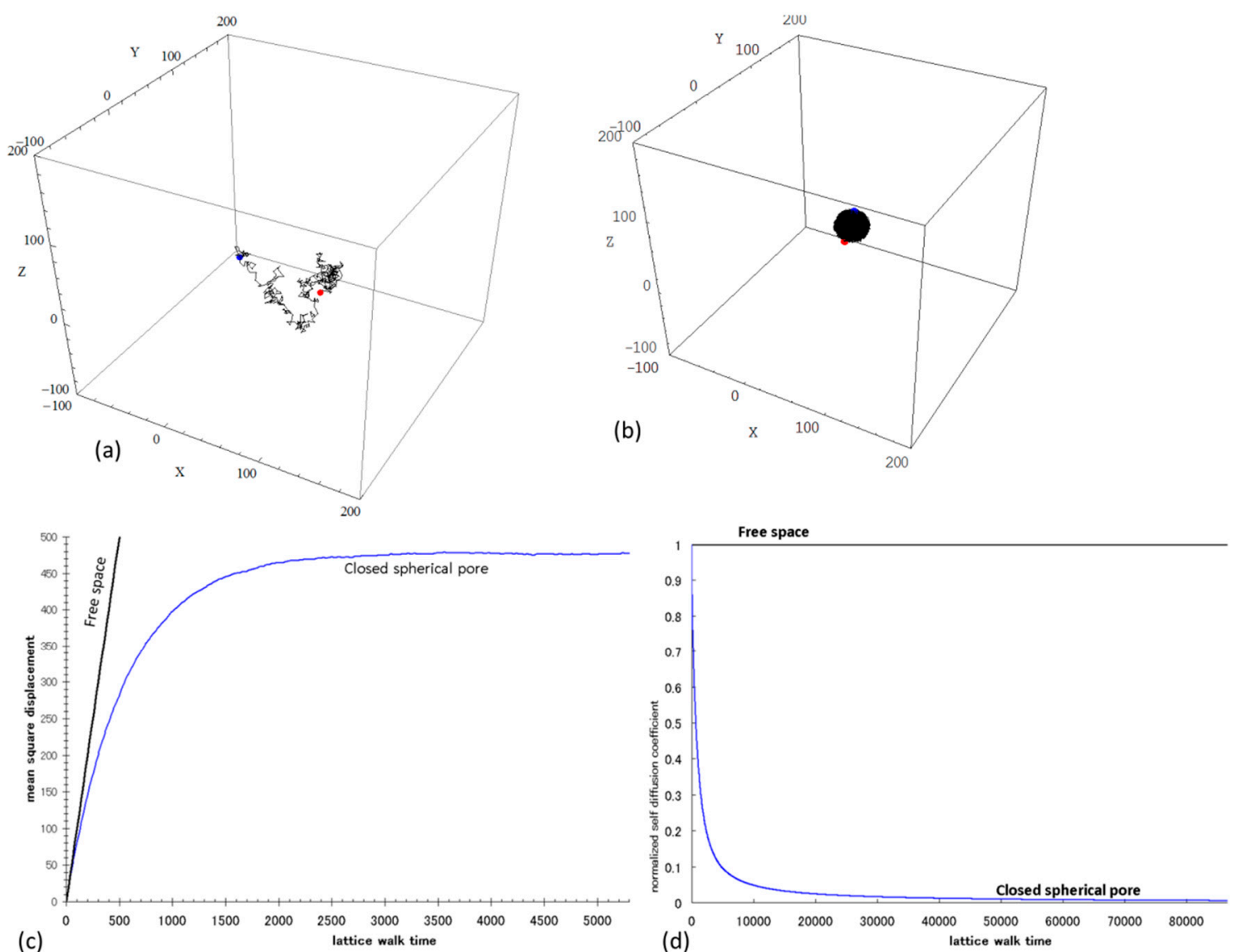

Figure 4. Sample output from the 3D Random Walk Simulation: (a) 3D trajectory of a walker in free space; (b) 3D trajectory of a walker in closed and isolated spherical pore; (c) plot of mean square displacement $\left(\langle r\rangle^{2}\right)$ vs. lattice walk time; (d) plot of the normalized self-diffusion coefficient $(D(t)) v s$. lattice walk time.
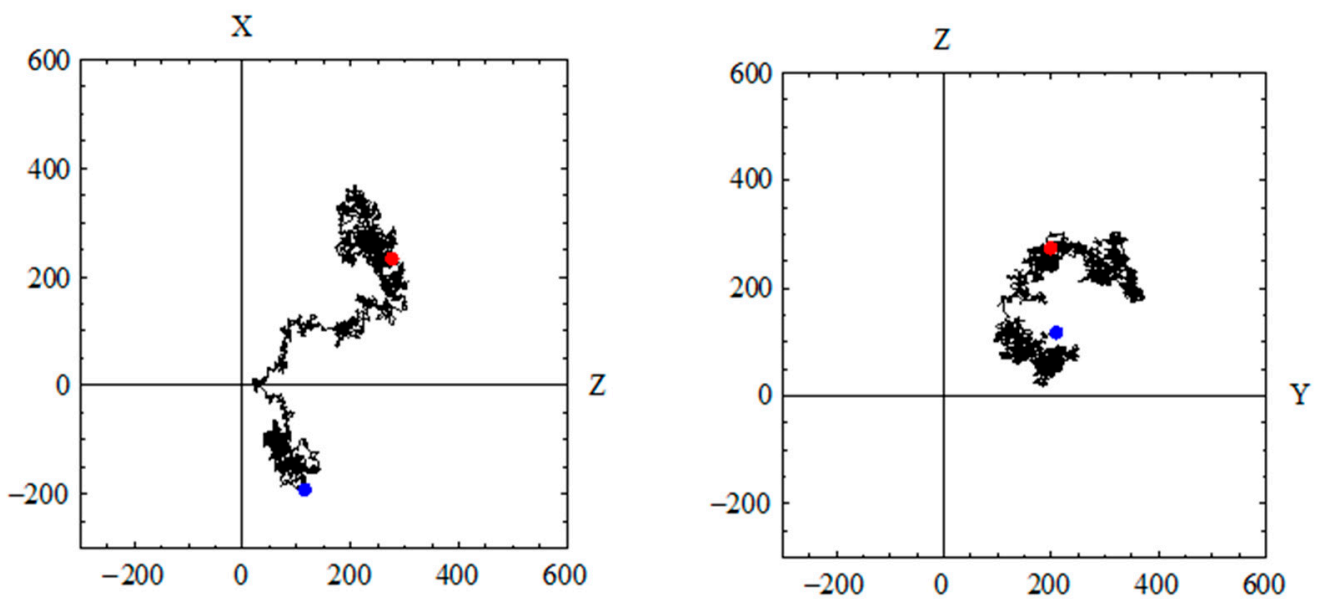

Figure 5. Cont. 


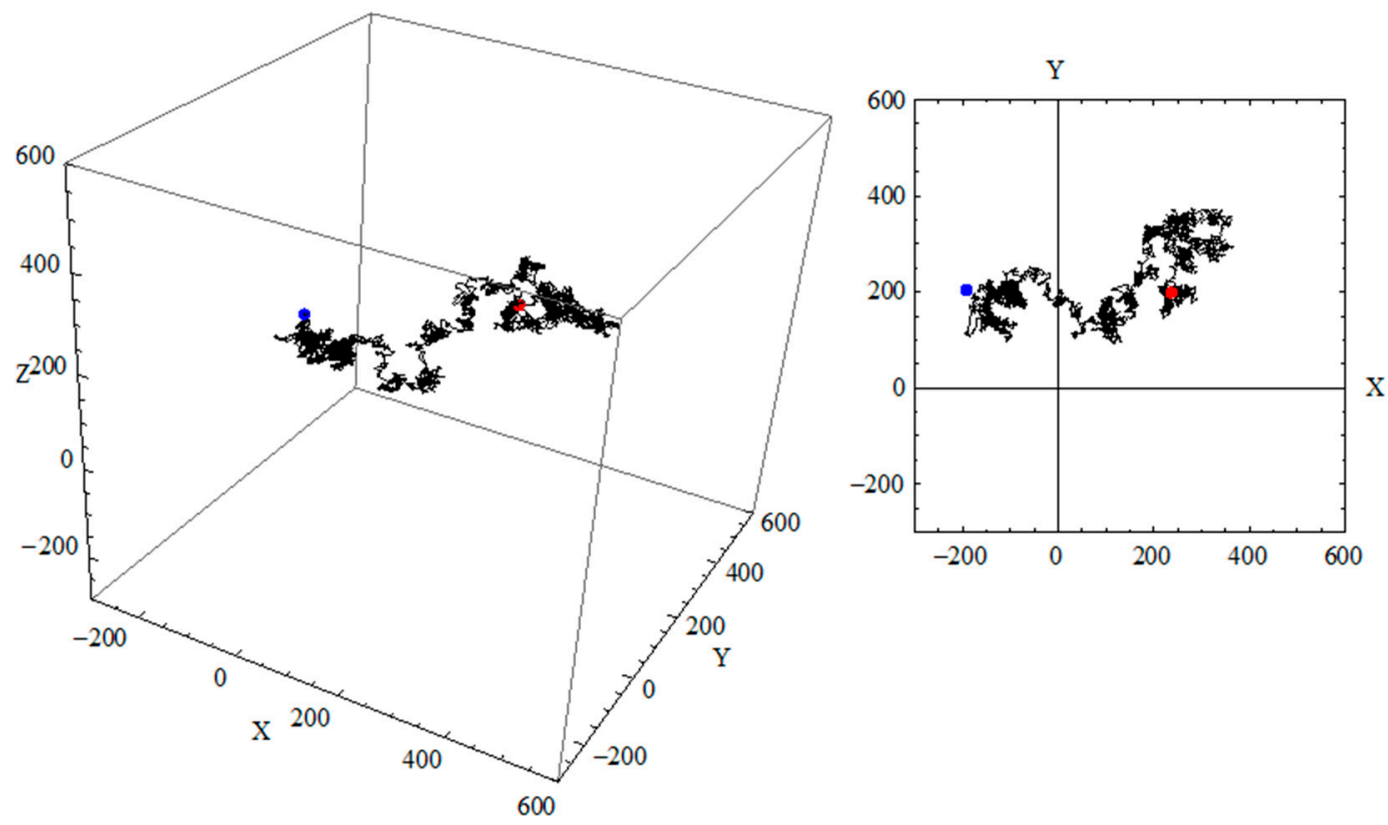

Figure 5. Sample trajectory of a walker in a digitized pore network of deteriorated cement paste including the projected trajectory in three orthogonal planes.

\subsubsection{Permeability}

The intrinsic permeability $K$, which is expressed in $\mathrm{m}^{2}$, characterizes the porous medium from the perspective of pressure-induced fluid flow through the fully saturated porosity. This parameter is theoretically the property of the medium and is independent of the penetrating fluid and applied pressure. On the other hand, hydraulic conductivity, also known as non-intrinsic permeability, is fluid-dependent and is expressed in $\mathrm{m} / \mathrm{s}$. The relationship between intrinsic permeability and hydraulic conductivity is shown by the following equation:

$$
\frac{K}{k_{h}}=\frac{\mu}{\rho g}
$$

where $\mu$ and $\rho$ are the fluid absolute viscosity and density.

The permeabilities of the percolated pore space in the VOI were computed using the NIST Stokes permeability solver [44]. Applications of this solver have been demonstrated in other porous materials [45,46]. The program is a 3D linear Stokes solver that performs calculations on 3D microstructures consisting of pores and solids. A pressure gradient ( 1 unit per voxel) is applied in one of the three orthogonal directions $(x, y$ or $z$ ). The program then calculates the resultant fluid velocity vector field within each pore voxel for slow, incompressible steady-state fluid flow by a finite difference solution for the linear Stokes equations. When this finite difference solution converges sufficiently, the intrinsic permeability of the porous medium is calculated by volume-averaging the local fluid velocity in the direction of flow using the Darcy equation:

$$
u=-\frac{K}{\mu} \frac{\Delta P}{L}
$$

where $u$ is the average fluid velocity in the direction of the flow, and $L$ is the length of the porous medium across which the pressure $\Delta P$ is applied. To obtain the permeabilities in all three directions, three separate runs of the computer programs on the 3D dataset were executed by changing the direction of flow. The average of the values corresponding to three directions was taken as the predicted permeability for a particular VOI. 
Note that the validation of the codes was first done using images generated in Image to model a simple pore structure in VOI of $300^{3}$ voxels, e.g., with 10 parallel cylindrical tubes of equal diameter as shown in Figure 6. Percentage errors between the theoretical permeability values and the simulated values were found to be very small, i.e., less than $3 \%$.

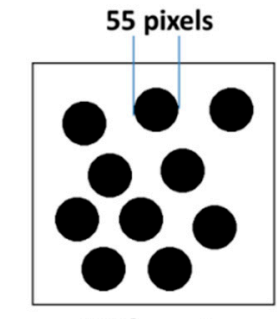

$300^{3}$ voxels

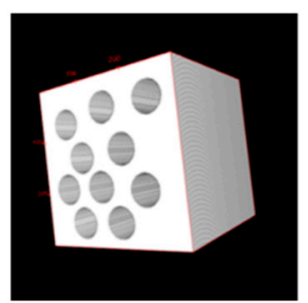

Computed permeability from NIST Stokes Permeability Solver $=24.8$ pixel $^{2}$

Theoretical permeability given the porosity $(\varnothing)$ and tube radius $(r)$ is:

$$
K=\phi \frac{r^{2}}{8}=\left(\frac{7,131,000}{27,000,000}\right) \frac{(55 / 2)^{2}}{8}=25.0 \text { pixels }^{2}
$$

Figure 6. Sample model pore structure used to validate the Stokes permeability solver.

\section{Results and Discussion}

Figure 7 summarizes the results of the analysis for the 5 samples representing the different regions of specimens exposed to accelerated leaching test. It can be observed that the most porous specimen was from OPC_de1 since this is the region where the surface was mostly exposed to water and also nearest to the cathode side of the accelerated leaching test. In this region, the effective porosity $\left(\phi_{\mathrm{e}}\right)$ of the deteriorated cement paste ranges from 0.28 to 0.33 . This is consistent with previous work reported in [16] wherein the reported effective porosity is 0.31 and 0.38 from the CT image of deteriorated cement paste and mortar, respectively. As expected, OPC_de2 was less porous $\left(\phi_{\mathrm{e}}=0.04-0.21\right)$ than OPC_de1, and the least porous was OPC_de3 $\left(\phi_{\mathrm{e}}=0.05-0.13\right)$ which is located at the center of the specimen. The other end of the specimen wherein the surface was also exposed to water, i.e., OPC_de5 was also found to be the second most porous microstructure with an effective porosity ranging from 0.13 to 0.18 . Although OPC_de5 was also exposed to water, the OPC_de1 is more deteriorated since the movement of calcium ions toward the cathode side results in an increased dissolution rate of calcium hydroxide and other hydrated cement products in the cement matrix. Such dissolution would result in increased porosity and pore size. This also results in increased permeability in both OPC_de1 and OPC_de5 as compared to OPC_de3, which has the lowest permeability.

Figures 8-10 describe the relationship between effective porosity and tortuosity, effective porosity and permeability, and tortuosity and permeability, respectively. Indications suggest that the more porous the material is, the less tortuous the transport of fluid is, which leads to a more permeable material. The permeability was found to be highly correlated with effective porosity and tortuosity, respectively, which is also consistent with the results presented in [18]. 


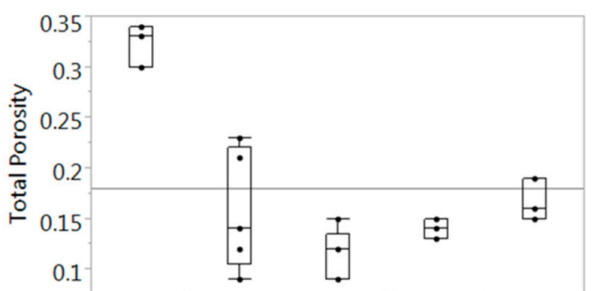

(a) OPC_de1 OPC_de2 OPC_de3 OPC_de4 OPC_de5 OPC

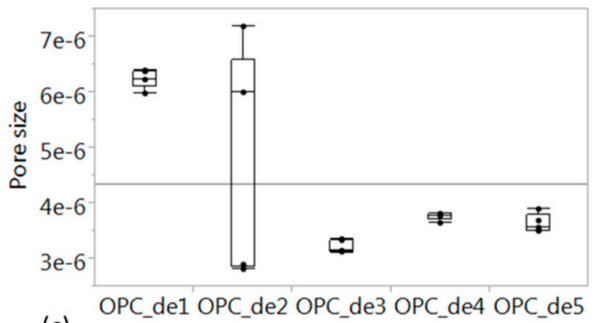

OPC

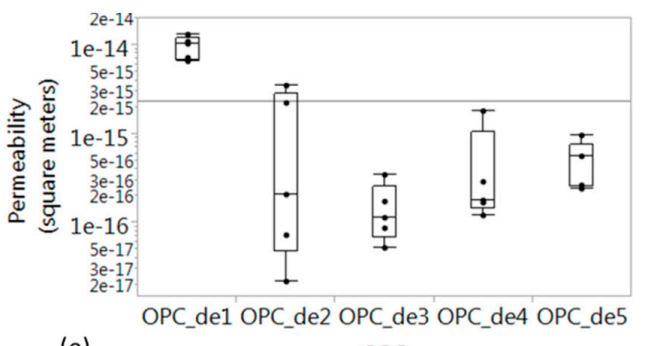

(e)

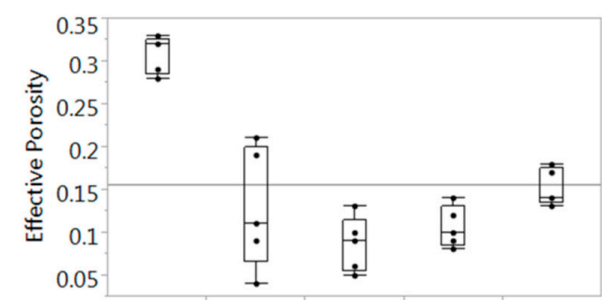

(b) OPC_de1 OPC_de2 OPC_de3 OPC_de4 OPC_de5 OPC

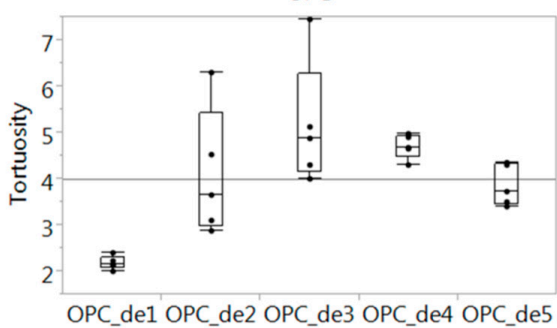

(d) OPC

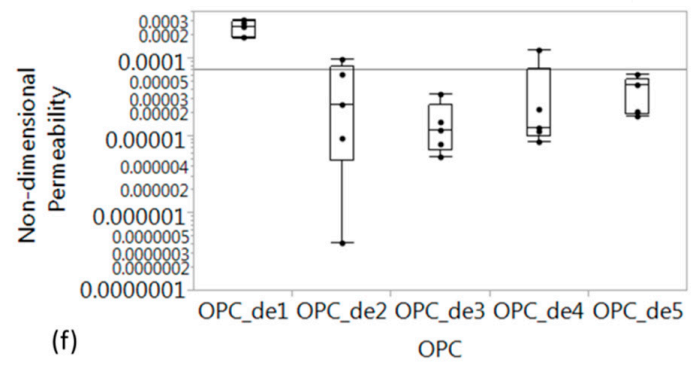

Figure 7. Summary of microstructure and transport properties in different regions: (a) Segmented total porosity; (b) effective porosity; (c) mean pore size (m); (d) tortuosity; (e) intrinsic permeability $\left(\mathrm{m}^{2}\right) ;(\mathbf{f})$ Non-dimensional permeability, i.e., intrinsic permeability normalized by square of the mean pore size.

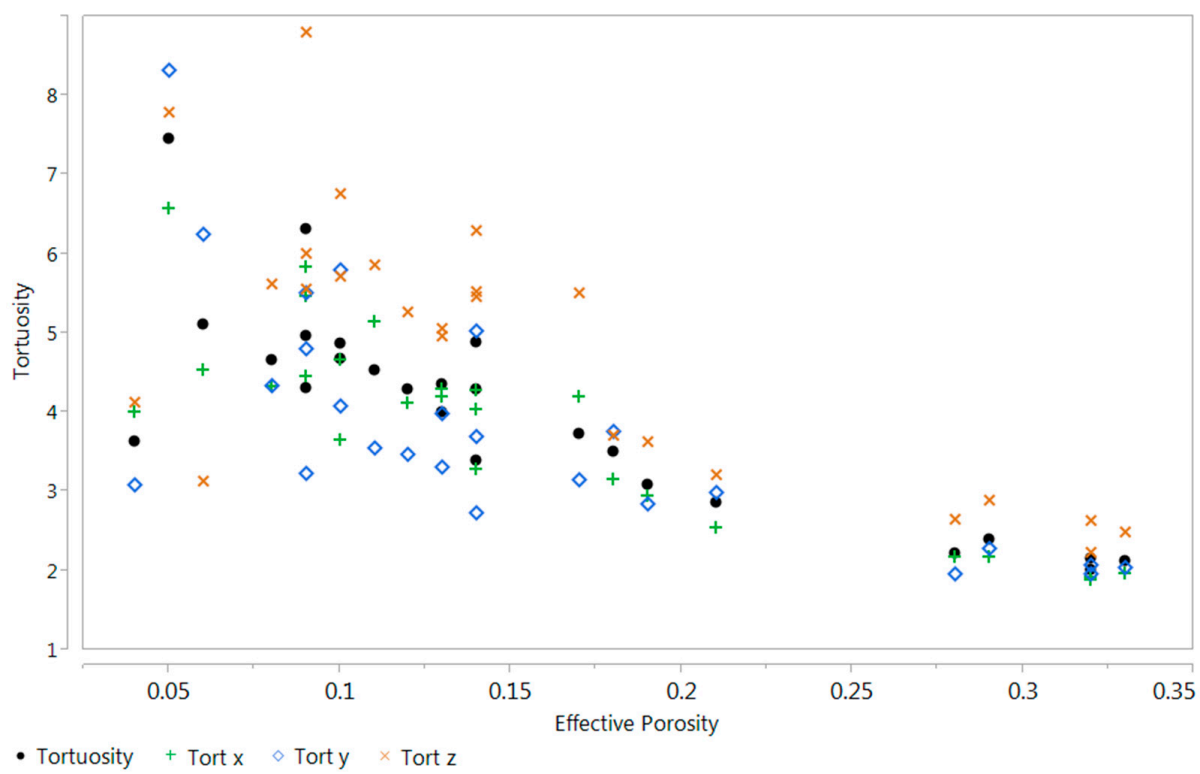

Figure 8. Scatter plot between effective porosity and tortuosity. 


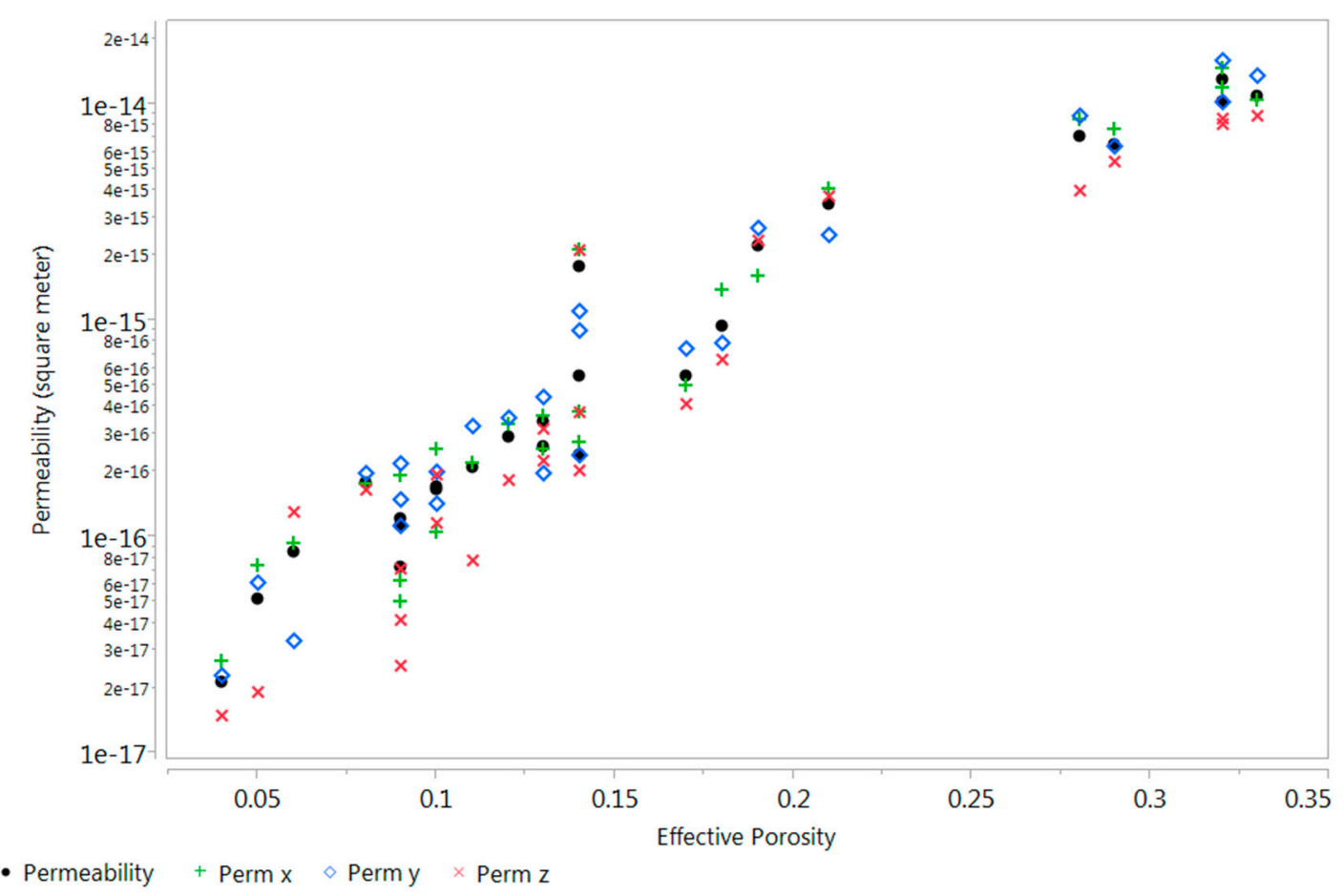

Figure 9. Scatter plot between effective porosity and intrinsic permeability $\left(\mathrm{m}^{2}\right)$.

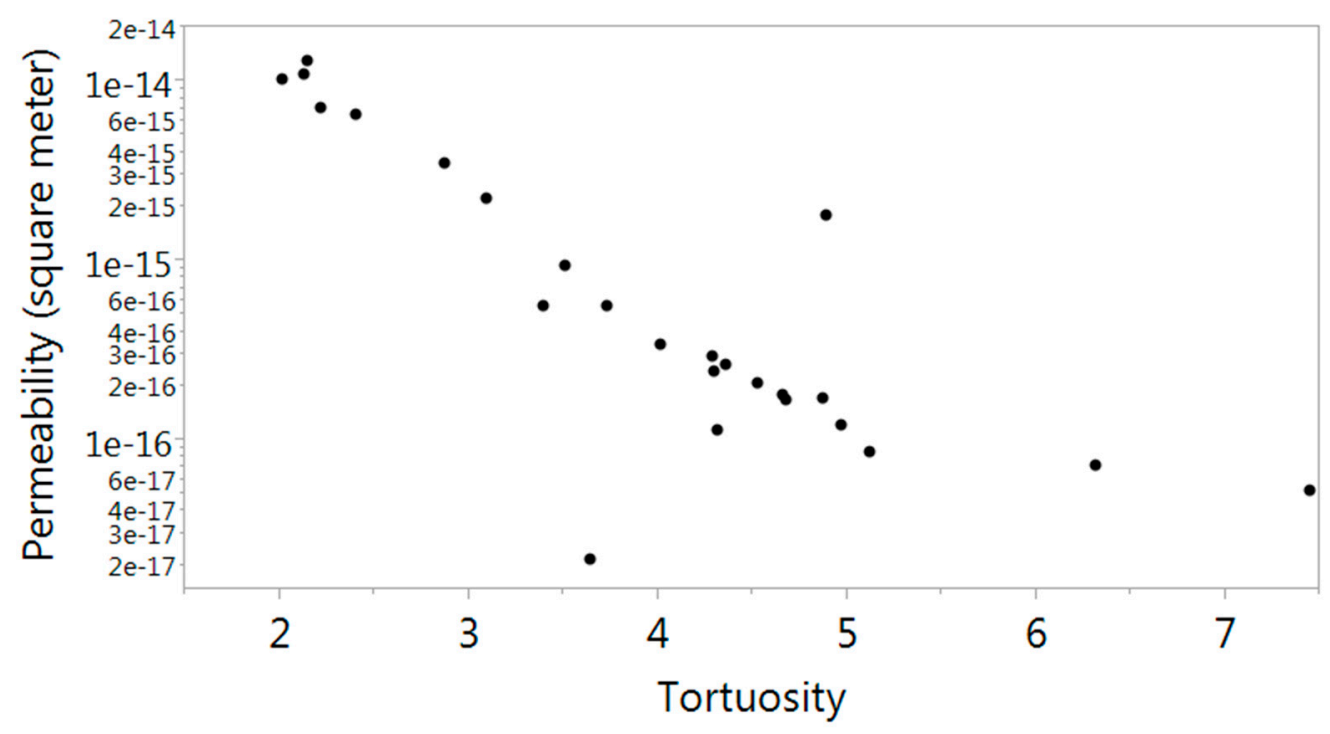

Figure 10. Scatter plot between tortuosity and intrinsic permeability $\left(\mathrm{m}^{2}\right)$.

Moreover, the microstructure-transport property correlation can be modeled as a power law function according to:

$$
\frac{K}{d^{2}}=a \phi_{e}^{b} \tau_{g}^{c}
$$

where $a, b$ and $c$ are the fitting parameters for this non-dimensional equation. Note the $K / d^{2}$ can be interpreted as the dimensionless permeability which was normalized by the square of the characteristic length scale of the pore structure. In this study, the mean pore size $(d)$ was used as the characteristic length scale. 
Table 1 summarizes the results of the non-linear regression analysis using one-, two-, and three-parameter fit for the non-dimensional permeability correlation. The correlation coefficient $(r)$ and the root mean square error (RMSE) were also given to describe the quality of the fit. Indication suggests that the two-parameter fit lead to a better predictive model than one-parameter fit particularly with the inclusion of both effective porosity and tortuosity parameter in the non-dimensional permeability correlation. On the other hand, the three-parameter fit leads only to marginal improvement in terms of the quality of fit. Thus, the microstructure-transport property correlation can be described by the two-parameter fit $K / d^{2}=0.004\left(\phi_{e} / \tau_{g}\right)^{1.42}$ as shown in Figure 11. This trend agrees with the experimental data described in [47], which suggests the effective porosity-tortuosity ratio as an alternative indicator for assessing concrete durability. Future studies would require validation of such non-dimensional transport model derived from CT images of deteriorated cement paste through additional experimental data. This work could also be extended to other characteristic length scales such as hydraulic diameter and critical pore diameter, and to other transport properties such as formation factor and ionic diffusivity.

Table 1. Fitting of the power law function $K / d^{2}=a \phi_{e}{ }^{b} \tau_{g}^{c}$ for the non-dimensional permeability correlation.

\begin{tabular}{ccccccc}
\hline Model & $\boldsymbol{a}$ & $\boldsymbol{b}$ & $\boldsymbol{c}$ & $\boldsymbol{r}^{\mathbf{1}}$ & RMSE $^{\mathbf{1}}$ & $\begin{array}{c}\text { Fitting } \\
\text { Parameter }\end{array}$ \\
\hline$K / d^{2}=a \phi_{e} \tau_{g}^{-1}$ & 0.0017 & 1 & -1 & 0.929 & $3.61 \times 10^{-5}$ & $\mathrm{a}$ \\
$K / d^{2}=a \phi_{e}^{b}$ & 0.0044 & 2.44 & 0 & 0.933 & $3.54 \times 10^{-5}$ & $\mathrm{a}, \mathrm{b}$ \\
$K / d^{2}=a \tau_{g}^{c}$ & 0.0026 & 0 & -3.17 & 0.917 & $3.92 \times 10^{-5}$ & $\mathrm{a}, \mathrm{c}$ \\
$K / d^{2}=a \phi_{e}^{b} \tau_{g}^{-1}$ & 0.0040 & 1.71 & -1 & 0.950 & $3.05 \times 10^{-5}$ & $\mathrm{a}, \mathrm{b}$ \\
$K / d^{2}=a \phi_{e} \tau_{g}^{c}$ & 0.0033 & 1 & -1.81 & 0.949 & $3.09 \times 10^{-5}$ & $\mathrm{a}, \mathrm{c}$ \\
$K / d^{2}=a \phi_{e}^{b} \tau_{g}^{-b}$ & 0.0040 & 1.42 & $(-\mathrm{b})$ & 0.952 & $3.01 \times 10^{-5}$ & $\mathrm{a}, \mathrm{b}$ \\
$K / d^{2}=a \phi_{e}^{b} \tau_{g}^{c}$ & 0.0040 & 1.43 & -1.36 & 0.952 & $3.03 \times 10^{-5}$ & a,b,c \\
\hline \multicolumn{7}{r}{$r=$ correlation coefficient, RMSE = root mean square (residual) error. }
\end{tabular}

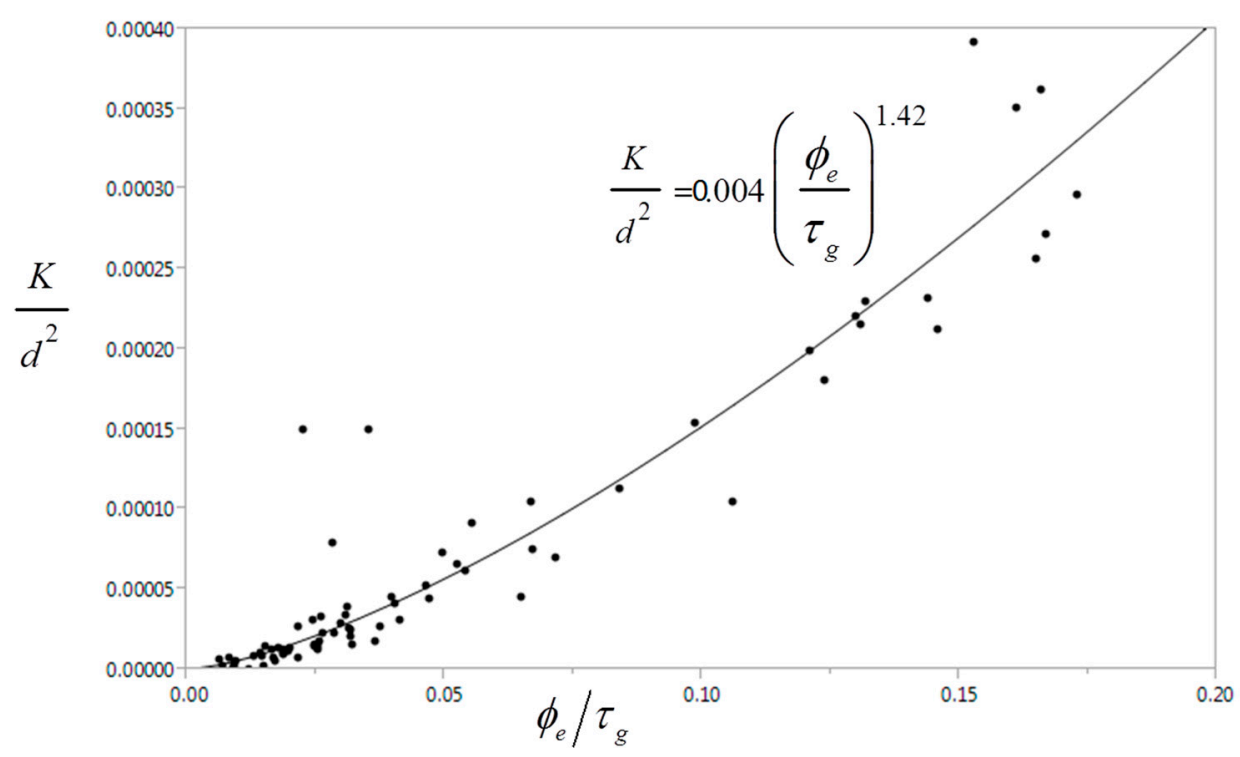

Figure 11. A non-dimensional model for microstructure-transport property correlation.

It should be noted that such an equation is a semi-empirical model analogous to permeability models for porous media such as that of the Kozeny-Carman or Katz-Thompson equations. It is physically meaningful as both the effective porosity and pore connectivity (characteristic pore size and 
pore network tortuosity) have been incorporated as a "permeability determiner". Such an approach is more meaningful than that of purely empirical analysis deduced from regression, and has been supported by a recently published work [48]. On the other hand, some open issues may still remain on whether the scale at the spatial resolution of CT image could measure the pore structure parameters relevant to the transport properties of deteriorated cement pastes. The current image resolution of X-ray microtomography may not be enough to distinguish those capillary pores, particularly at the sub-micron and nanometer scale. The calculated values may deviate from the measured permeability in deteriorated cement pastes as pores smaller than the image resolution are not considered in the simulation. Nevertheless, the coarser pore size resolved from the CT images in this study are attributed mainly to portlandite dissolution, which could be the controlling factor in the permeability of such deteriorated cement pastes, but this requires further investigation. Moreover, it has also been suggested that the self-similarity of the capillary pores resolved from CT images can be used to provide the same pore space morphology with that of the pore network relevant to transport [49]. Other complementing methods such as that of simulated microstructure and instrumental analysis could give a more realistic pore structure [50] or some correction for the unresolved sub-micron features [27] in the apparently poor pore network connectivity reported in cement pastes, e.g., with low water-to-cement ratio or pastes containing silica fume. The up-scaling across a range of length scales will also be a critical issue for predicting the transport properties not only in deteriorated cement pastes, but also in mortar and concrete. Thus, a multi-scale modeling scheme, which is analogous to what has been applied to undamaged cementitious material $[19,42,51]$, will be appropriate to address this kind of issue.

\section{Conclusions}

X-ray microtomography allows us to visualize the microstructure of leaching-induced deteriorated cement paste in three dimensions. Coupled with 3D image analysis and numerical simulation in the digitized pore space, microstructure and transport properties such as effective porosity, pore size, tortuosity and permeability were computed. Computation of these parameters as characteristic material constants for a porous medium provides an opportunity to derive microstructure-transport property correlation across three orders of magnitude in permeability. Indication suggests that the effective porosity-tortuosity ratio could be used as an indicator of transport properties of deteriorated cement pastes.

Acknowledgments: This research was partially supported by the DLSU URCO-funded faculty research grant (Project No. 44 F U 3 11).

Author Contributions: Michael Angelo B. Promentilla and Takafumi Sugiyama conceived and designed the methodology including the write-up of the main narrative of the paper; Shermaine M. Cortez, Regina Anne DC. Papel and Bernadette M. Tablada helped in performing the simulation experiments and analyzing the data.

Conflicts of Interest: The authors declare no conflict of interest.

\section{References}

1. Bentur, A. Cementitious materials-Nine millennia and a new century: Past, present, and future. J. Mater. Civ. Eng. 2002, 14, 2-22. [CrossRef]

2. Mehta, P.; Monteiro, P. Concrete: Microstructure, Properties, and Materials; McGraw-Hill Companies: New York, NY, USA, 2006.

3. Basheer, L.; Kropp, J.; Cleland, D. Assessment of the durability of concrete from its permeation properties: A review. Constr. Build. Mater. 2001, 15, 93-103. [CrossRef]

4. Gallé, C.; Peycelon, H.; Le Bescop, P. Effect of an accelerated chemical degradation on water permeability and pore structure of cement-based materials. Adv. Cem. Res. 2004, 16, 105-114. [CrossRef]

5. Carde, C.; Francois, R. Effect of the leaching of calcium hydroxide from cement paste on mechanical and physical properties. Cem. Concr. Res. 1997, 274, 539-550. [CrossRef] 
6. Lin, W.; Cheng, A.; Huang, R.; Chen, C.; Zhou, X. Effect of Calcium Leaching on the Properties of Cement based Composites. J. Wuhan Univ. Technol. Mater. Sci. Ed. 2011, 26, 990-997. [CrossRef]

7. Adenot, F.; Buil, M. Modelling of the corrosion of the cement paste by deionized water. Cem. Concr. Res. 1992, 22, 489-496. [CrossRef]

8. Mainguy, M.; Tognazzi, C.; Torrenti, J.M.; Adenot, F. Modelling of leaching in pure cement paste and mortar. Cem. Concr. Res. 2000, 30, 83-90. [CrossRef]

9. Ryu, J.S.; Otsuki, N.; Minagawa, H. Long-term forecast of Ca leaching from mortar and associated degeneration. Cem. Concr. Res. 2002, 32, 15-39. [CrossRef]

10. Saito, H.; Deguchi, A. Leaching tests on different mortars using accelerated electrochemical method. Cem. Concr. Res. 2000, 30, 1815-1825. [CrossRef]

11. Saito, H.; Nakane, S.; Ikari, S.; Fujiwara, A. Preliminary experimental study on the deterioration of cementations materials by an acceleration method. Nucl. Eng. Des. 1992, 138, 151-155. [CrossRef]

12. Stora, E.; Bary, B.; He, Q.-C.; Deville, E.; Montarnal, P. Modelling and simulations of the chemo-mechanical behaviour of leached cement-based materials: Leaching process and induced loss of stiffness. Cem. Concr. Res. 2009, 39, 763-772. [CrossRef]

13. Bernard, F.; Kamali-Bernard, S. Performance simulation and quantitative analysis of cement-based materials subjected to leaching. Comput. Mater. Sci. 2010, 50, 218-226. [CrossRef]

14. Yu, Y.; Zhang, Y.X.; Khennane, A. Numerical modelling of degradation of cement-based materials under leaching and external sulfate attack. Comput. Struct. 2015, 158, 1-14. [CrossRef]

15. Wan, K.; Lib, Y.; Sun, W. Experimental and modelling research of the accelerated calcium leaching of cement paste in ammonium nitrate solution. Constr. Build. Mater. 2013, 40, 832-846. [CrossRef]

16. Sugiyama, T.; Promentilla, M.A.B.; Hitomi, T.; Takeda, N. Application of synchrotron microtomography for pore structure characterization of deteriorated cementitious materials due to leaching. Cem. Concr. Res. 2010, 40, 1265-1270. [CrossRef]

17. Burlion, N.; Bernard, D.; Chen, D. X-ray microtomography: Application to microstructure analysis of a cementitious material during leaching process. Cem. Concr. Res. 2006, 36, 346-357. [CrossRef]

18. Zhang, M.; Ye, G.; van Breugel, K. Microstructure-based modeling of permeability of cementitious materials using multiple-relaxation-time lattice Boltzmann method. Comput. Mater. Sci. 2013, 68, 142-151. [CrossRef]

19. Zhang, M.; Ye, G.; van Breugel, K. Multiscale lattice Boltzmann-finite element modelling of chloride diffusivity in cementitious materials. Part II: Simulation results and validation. Mech. Res. Commun. 2014, 58, 64-72. [CrossRef]

20. Wong, H.S.; Zimmerman, R.; Buenfeld, N. Estimating the permeability of cement pastes and mortars using image analysis and effective medium theory. Cem. Concr. Res. 2012, 42, 476-483. [CrossRef]

21. Wong, H.S.; Buenfeld, N.R.; Head, M.K. Estimating transport properties of mortars using image analysis on backscattered electron images. Cem. Concr. Res. 2006, 36, 1556-1566. [CrossRef]

22. Promentilla, M.A.B.; Sugiyama, T.; Hitomi, T.; Takeda, N. Quantification of tortuosity in hardened cement pastes using synchrotron-based X-ray computed microtomography. Cem. Concr. Res. 2009, 39, 548-557. [CrossRef]

23. Promentilla, M.A.B.; Sugiyama, T.; Hitomi, T.; Takeda, N. Characterizing the 3D pore structure of hardened cement paste with synchrotron microtomography. J. Adv. Concr. Technol. 2008, 6, 273-286. [CrossRef]

24. Hu, J.; Stroeven, P. Local porosity analysis of pore structure in cement paste. Cem. Concr. Res. 2005, 35, 233-242. [CrossRef]

25. Bentz, D.P. Quantitative Comparison of Real and CEMHYD3D Model Microstructures Using Correlation Functions. Cem. Concr. Res. 2006, 36, 259-263. [CrossRef]

26. Zhang, M.; He, Y.; Ye, G.; Lange, D.; van Breugel, K. Computational investigation on mass diffusivity in Portland cement paste based on X-ray computed microtomography (CT) image. Constr. Build. Mater. 2012, 27, 472-481. [CrossRef]

27. Karim, M.; Krabbenhoft, K. Extraction of Effective Cement Paste Diffusivities from X-ray Microtomography Scans. Transp. Porous Media 2010, 84, 371-388. [CrossRef]

28. Stora, E.; Bary, B.; He, Q.C.; Deville, E.; Montarna, P. Modelling and simulations of the chemo-mechanical behaviour of leached cement-based materials: Interactions between damage and leaching. Cem. Concr. Res. 2010, 40, 1226-1236. [CrossRef] 
29. Zhang, M.; Jivkov, A. Microstructure-informed modelling of damage evolution in cement paste. Constr. Build. Mater. 2014, 66, 731-742. [CrossRef]

30. Bangert, F.; Grasberger, S.; Kuhl, D.; Meschke, G. Environmentally induced deterioration of concrete: Physical motivation and numerical modelling. Eng. Fract. Mech. 2003, 70, 891-910. [CrossRef]

31. Wong, H.S.; Head, M.K.; Buenfeld, N.R. Pore segmentation of cement-based materials from backscattered electron images. Cem. Concr. Res. 2006, 36, 1083-1090. [CrossRef]

32. Hoshen, J.; Kopelman, R. Percolation and cluster distribution. I. Cluster multiple labeling technique and critical concentration algorithm. Phys. Rev. B 1976, 14, 34-38. [CrossRef]

33. Rasband, W. ImageJ: Image Processing and Analysis in Java, National Health Institute (NIH). Available online: http:/ /rsb.info.nih.gov/ij/ (accessed on 16 May 2016).

34. Nakano, T.; Tsuchiyama, A.; Uesugi, K.; Uesugi, M.; Shinohara, K. SLICE-Software for basic 3-D image analysis. Available online: http://www-bl20.spring8.or.jp/slice/ (accessed on 16 May 2016).

35. Doube, M.; Kosowski, M.; Arganda-Carreras, I.; Cordelières, F.; Dougherty, R.P.; Jackson, J.; Schmid, B.; Hutchinson, J.R.; Shefelbine, S.J. BoneJ: Free and extensible bone image analysis in ImageJ. Bone 2010, 47, 1076-1079. [CrossRef] [PubMed]

36. Promentilla, M.A.; Sugiyama, T. X-ray Microtomography of Mortars Exposed to Freezing-Thawing Action. J. Adv. Concr. Technol. 2010, 8, 97-111. [CrossRef]

37. Hildebrand, T.; Ruegsegger, P. A new method for the model-independent assessment of thickness in three-dimensional images. J. Microsc. 1997, 185, 67-75. [CrossRef]

38. Dougherty, R.; Kunzelmann, K. Computing local thickness of 3D structures with ImageJ. Microsc. Microanal. 2007, 13, 1678-1679. [CrossRef]

39. Promentilla, M.A.; Sugiyama, T. Application of Microfocus X-ray CT to Investigate the Frost-Induced Damage Process in Cement-Based Materials. In Advances in Computed Tomography for Geomaterials: GeoX 2010; Alshibli, K.A., Reed, A.H., Eds.; John Wiley \& Sons, Inc.: Hoboken, NJ, USA, 2010.

40. Darma, I.S.; Sugiyama, T.; Promentilla, M.A.B. X-ray CT to study diffusivity in cracked concrete through the observation of tracer transport. J. Adv. Concr. Technol. 2013, 11, 266-281. [CrossRef]

41. Nakashima, Y.; Kamiya, S. Mathematica programs for the analysis of three-dimensional pore connectivity and anisotropic tortuosity of porous rocks using X-ray microtomography. J. Nucl. Sci. Technol. 2007, 44, 1233-1247. [CrossRef]

42. Ma, H.; Hou, D.; Li, Z. Two-scale modeling of transport properties of cement paste: Formation factor, electrical conductivity and chloride diffusivity. Comput. Mater. Sci. 2015, 110, 270-280. [CrossRef]

43. Stauffer, D.; Aharony, A. Introduction to Percolation Theory, Revised; Taylor and Francis: London, UK, 1994.

44. Bentz, D.; Martys, N. A Stokes Permeability Solver for Three Dimensional Porous Media. Available online: http:/ / concrete.nist.gov/ \{\}bentz/NISTIR7416.pdf (accessed on 16 May 2016).

45. Sun, X.; Daia, Q.; Ng, K. Computational investigation of pore permeability and connectivity from transmission X-ray microscope images of a cement paste specimen. Constr. Build. Mater. 2014, 68, 240-251.

46. Bentz, D.P. Virtual pervious concrete: Microstructure, percolation, and permeability. ACI Mater. J. 2008, 105, 297-301.

47. Ahmad, S.; Azad, A.K. An exploratory study on correlating the permeability of concrete with its porosity and tortuosity. Adv. Cem. Res. 2013, 25, 288-294. [CrossRef]

48. Ma, H.; Xu, B.; Liu, J.; Pei, H.; Li, Z. Effects of water content, magnesia-to-phosphate molar ratio and age on pore structure, strength and permeability of magnesium potassium phosphate cement paste. Mater. Des. 2014, 64, 497-502. [CrossRef]

49. Koster, M.; Hannawald, J.; Brameshuber, W. Simulation of water permeability and water vapor diffusion through hardened cement paste. Comput. Mech. 2006, 37, 163-172. [CrossRef]

50. Ma, H.; Li, Z. Realistic pore structure of Portland cement paste: Experimental study and numerical simulation. Comput. Concr. 2013, 11, 317-336. [CrossRef]

51. Ma, H.; Hou, D.; Lu, Y.; Li, Z. Two-scale modeling of the capillary network in hydrated cement paste. Constr. Build. Mater. 2014, 64, 11-21. [CrossRef]

(C) 2016 by the authors; licensee MDPI, Basel, Switzerland. This article is an open access article distributed under the terms and conditions of the Creative Commons Attribution (CC-BY) license (http://creativecommons.org/licenses/by/4.0/). 